\title{
Serge Rombouts, Frederik Barkhof, Philip Scheltens (eds): Clinical application of functional brain MRI
}

\author{
Oxford University Press, 2007, 386 pages, HB, ISBN 978-0-19-856629-8, \\ 125.00 (GBP Sterling)
}

\author{
David Brennan • Donald M. Hadley
}

Received: 8 December 2008 / Accepted: 22 December 2008 / Published online: 29 January 2009

(C) Springer-Verlag 2009

Keywords fMRI · Schizophrenia · Parkinson's disease ·

Dementia $\cdot$ Depression stroke

With increasing numbers of clinicians becoming interested in the benefits of functional magnetic resonance imaging (fMRI), there is certainly a need for good literature on the use of fMRI in clinical applications.

The title of the book suggests that within its pages it contains details on the theory, application, and practicalities of clinical fMRI. One would also expect it to deal with the issues of reproducibility, errors, and limitations. In fact, the first three chapters and to a lesser extent chapter 4 (148 pages) do very good job covering these issues. They do not, however, provide any specific practical advice on the use of fMRI statistical packages. You would have to look elsewhere for advice and explanation on their selection and implementation.

The early chapters focus on motor, language, and memory fMRI paradigms, which are the staple of clinical fMRI. They describe in reasonable detail why fMRI is required, which functional regions of the cortex are involved, what methodologies should be used, what the limitations are, and what the advantages and disadvantages

\section{Brennan $(\bowtie)$}

Department of Clinical Physics, Institute of Neurological

Sciences, Southern General Hospital,

1345 Govan Road,

Glasgow G51 4TF, UK

e-mail: d.brennan@clinmed.gla.ac.uk

D. M. Hadley

Department of Neuroradiology, Institute of Neurological Sciences,

Southern General Hospital,

Glasgow, UK are in using fMRI compared to other modalities such as PET. Surprisingly, there is no section on visual activation paradigms that I would have expected to be covered in this type of text.

The remaining eight chapters (231 pages) focused on specific diseases such as dementia, schizophrenia, depression, and stroke, with the chapter on depression being the most detailed and thorough. However, contrary to what the title of the book suggests, it does not describe the clinical use of fMRI in these conditions. It actually describes the research that has been conducted into these conditions using fMRI. Despite this deviation from the main theme of the book, the chapters here are an interesting read and a good start if you are interested in research in these areas. The chapter on Parkinson's disease is a little weak and short, although this may partly reflect a lack of research in the area rather than a poorly written chapter.

So, in effect, there are two distinct sections within this book. The first describes the clinical use of fMRI, and the second describes research in areas where fMRI might be applicable in the clinic at some point in the future. Overall, the writing is very good, however, as with any multi-author book, it can vary from chapter to chapter.

The volume would certainly find a place in a department library. Trainees in neuroradiology would learn a lot from dipping into it while established neuroradiologists and neuroscientists could gain insights into fMRI. Researchers would probably prefer to glean the same information from the many review papers of the individual subjects covered in this book.

If, in general, you are looking for a book that provides a good introduction into the use of clinical fMRI while also providing a good starting point for expanding into clinical research, then I would certainly recommend this book. 